\title{
Topical Issue on Giant, Pygmy, Pairing Resonances and Related Topics
}

\author{
A tribute to Pier Francesco Bortignon
}

Published online: 2 December 2019 - (c) Società Italiana di Fisica / Springer-Verlag GmbH Germany, part of Springer Nature, 2019

The present volume is, in keeping with its title, divided into four sections, in each of which one finds contributions from world specialists, covering a wide variety of subjects lying at the forefront of nuclear research.

Quoting from the first scientific contribution, the articles of the present special issue are dedicated to the memory of Pier Francesco Bortignon and devoted to developments related to his pioneering ideas. In this contribution, results of relativistic nuclear field theory at finite temperature, with special emphasis on damping phenomena are presented. These are subjects to which Pier Francesco dedicated much effort and provided equally much insight, especially concerning the role doorway states play in the damping of collective motion in general, and of giant resonances in particular. Also concerning the damping due to the decay into individual particle-hole excitations (Landau damping) discussed in detail in another contribution to the first section, in connection with the analysis of the gross, intermediate and fine structure of giant resonances.

Moving to the second section dealing with pygmy resonances, not surprisingly a very rich section, we are confronted, among many other contributions, with an overview of selected experimental work on the gamma-decay from the GDR and the PDR, which acknowledges inspiration provided by Pier Francesco through the years, in particular concerning the problem of isospin symmetry in nuclei at finite temperature and of pygmy states far from stability. Within this context, another experimental contribution which concentrates on light exotic halo nuclei refers, in connection with

${ }^{11} \mathrm{Li}$, to nuclear field theory calculations of Pier Francesco and collaborators, providing a simultaneous description of both the soft E1-mode (pygmy) and the strong mixing of $s$ - and $p$-waves in the ground state.

Coming to the third section, one reads about the observation of Giant Pairing Vibrations not in heavy, as expected, but in light nuclei. In connection with the ensuing elaboration of such a result, work of Pier Francesco is referred to in which a possible explanation is suggested, based on the much larger width expected for GPV in heavy nuclei as compared with light nuclei.

The last section, reflecting its title, is more inhomogeneous than the three previous ones. Nonetheless, there is a red thread going through almost all of the contributions, namely nuclear field theory, particle-vibration coupling mechanism and doorway damping, subjects which lay at the center of Pier Francesco interests, and to which he so extensively and eminently contributed.

Not only do the subjects in the different contributions honor the memory of Pier Francesco, but also the scientific and professional level with which they are treated constitutes a most proper tribute to the never-ceasing quest for accuracy which was characteristic of Pier Francesco's scientific endeavor.

N. Alamanos, R.A. Broglia, and E. Vigezzi 